\title{
Model independence in two dimensions and polarized cold dipolar molecules
}

\author{
A.G. Volosniev, D.V. Fedorov, A.S. Jensen, and N.T. Zinner \\ Department of Physics and Astronomy, Aarhus University, DK-8000 Aarhus C, Denmark
}

(Dated: June 8, 2018)

\begin{abstract}
We calculate the energy and wave functions of two particles confined to two spatial dimensions interacting via arbitrary anisotropic potentials with negative or zero net volume. The general rigorous analytic expressions are given in the weak coupling limit where universality or model independence are approached. The monopole part of anisotropic potentials is crucial in the universal limit. We illustrate the universality with a system of two arbitrarily polarized cold dipolar molecules in a bilayer. We discuss the transition to universality as function of polarization and binding energy, and compare analytic and numerical results obtained by the stochastic variational method. The universal limit is essentially reached for experimentally accessible strengths.
\end{abstract}

PACS numbers: 03.65.Ge,36.20.-r,67.85.-d,73.20.-r

Introduction. The existence of bound quantum states in a potential is a problem that appears in many scientific disciplines. While in three dimensions it is known that sufficiently shallow potentials will not bind, for one- and two-dimensional non-positive potentials early arguments by Landau and Lifshitz [1] demonstrate that at least one bound state is always present for arbitrarily small potential strength. Only much later this was generalized to potentials of negative or zero spatial volume by Simon [2]. Such bound states and their structures are not only of formal interest but serve a great number of applications in several areas including exotic atoms [3], excitons in carbon nanotubes [4] or semiconducting microcavities [5] and organic interfaces [6], pairing in two-dimensional Fermi gases 7], localization of adatoms on surfaces [8] or through nanosctructuring [9], and in population genetics [10].

In this letter we introduce a general formalism to calculate the leading, as well as higher, order terms for arbitrary anisotropic weak potentials. This opens the door for many new applications since physical systems usually have anisotropic features that make models assuming cylindrical symmetry inaccurate. We test our formulas in the emerging field of cold polar molecules [11] where layered geometries have recently been experimentally realized [12]. The long-range dipolar potential can be made anisotropic by tilting the external aligning field. Novel superfluid and density-wave instabilites have been proposed in this geometry [13]. However, very little is known about few-body bound states in the system, particularly in the experimentally relevant weak coupling limit, where universal features emerge independent of specific models, and previous works have only considered the case of perpendicular polarization [14 16]. We shall compare analytic and numerical results and investigate the approach towards the universal limit. We deduce some implications for three-body [17] as well as for many-body states in two dimensions (2D).

Formulation. Consider two particles confined to two spatial dimensions and interacting via a pair potential,
$V(\vec{r})$, where $\vec{r}$ is the relative coordinate. Using polar coordinates, $\vec{r}=(x, y)=(r \cos \varphi, r \sin \varphi)$, we can write the Schrödinger equation as

$$
\left[-\frac{1}{s} \frac{\partial}{\partial s} s \frac{\partial}{\partial s}-\frac{1}{s^{2}} \frac{\partial^{2}}{\partial \varphi^{2}}+\lambda \frac{2 \mu d^{2}}{\hbar^{2}} V(s, \varphi)\right] \psi=\alpha^{2} \psi,
$$

with $\psi$ the wave function, $\mu$ the reduced mass, $\lambda$ the dimensionless strength, $\alpha^{2}=2 \mu d^{2} E / \hbar^{2}, E$ the energy, $d$ the unit of length, and $s=r / d$ the reduced coordinate. The wave function can be partial-wave expanded,

$$
\psi(s, \varphi)=\frac{1}{\sqrt{s}} \sum_{m=-\infty}^{m=\infty} a_{m} f_{m}(s) \exp (i m \varphi)
$$

where $a_{m}$ are the expansion coefficients and the $2 \mathrm{D}$ radial wave functions, $f_{m}(s)$, satisfy the system of equations

$$
f_{m}^{\prime \prime}+\frac{1-4 m^{2}}{4 s^{2}} f_{m}+\alpha^{2} f_{m}=\lambda \sum_{l} \frac{a_{l}}{a_{m}} f_{l} V_{m l}(s) .
$$

The matrix elements, $V_{m l}$,

$$
V_{m l}=\frac{1}{2 \pi} \frac{2 \mu d^{2}}{\hbar^{2}} \int_{0}^{2 \pi} e^{i(l-m) \varphi} V(s, \varphi) \mathrm{d} \varphi,
$$

carry all information about the potential. For cylindrical potentials $V_{m l} \propto \delta_{m l}$ and the different $m$-values decouple in Eq. (3).

General derivation. The regular radial solution to Eq. (3) at the origin provides the usual boundary condition for a centrifugal barrier potential, i.e. we choose $\lim _{s \rightarrow 0} s^{-1 / 2-|m|} f_{m}(\alpha, s)=1$, where we inserted explicitly the dependence on the energy parameter, $\alpha$, in $f_{m}$. We assume that the potential, and consequently also the right hand side of Eq. (3), diverge slower than $1 / s^{2}$ when $s \rightarrow 0$. The integral form of the equations in (3) is given as in [18],

$$
\begin{aligned}
& a_{m} f_{m}(\alpha, s)=a_{m} F_{m}(\alpha, s) \\
& -\lambda \sum_{l} a_{l} \int_{0}^{s} g_{|m|}\left(\alpha, s, s^{\prime}\right) V_{m l}\left(s^{\prime}\right) f_{l}\left(\alpha, s^{\prime}\right) \mathrm{d} s^{\prime},
\end{aligned}
$$


where the boundary condition at $s=0$ is obeyed through the solution of the free Schrödinger equation:

$$
F_{m}(\alpha, s)=\sqrt{s} J_{|m|}(\alpha s)(2 / \alpha)^{|m|}|m| !,
$$

where $J_{m}$ is the Bessel function and where $g_{|m|}$ in Eq. (5) is the Green's function given as

$$
\begin{array}{r}
g_{|m|}\left(\alpha, s, s^{\prime}\right)=\frac{i \pi}{4} \sqrt{s s^{\prime}}\left[H_{|m|}^{(1)}(\alpha s) H_{|m|}^{(2)}\left(\alpha s^{\prime}\right)\right. \\
\left.-H_{|m|}^{(1)}\left(\alpha s^{\prime}\right) H_{|m|}^{(2)}(\alpha s)\right]
\end{array}
$$

in terms of the Hankel functions, $H_{m}^{(n)}$. For bound states (where $\alpha=i|\alpha|$ ) both the free wave functions in Eq. (6) and the Green's function in Eq. (7) diverge at large distances, but their combination in Eq. (5) must vanish exponentially. This provides the quantization condition for the energy. Mathematically the condition is given as

$$
\begin{gathered}
\sum c_{m l} a_{l}=-a_{m}, \\
c_{m l}= \\
\lambda\left(\frac{\alpha}{2}\right)^{|m|} \frac{i \pi}{2|m| !} \int_{0}^{\infty} \sqrt{s^{\prime}} H_{|m|}^{(1)}\left(\alpha s^{\prime}\right) V_{m l}\left(s^{\prime}\right) f_{l}\left(\alpha, s^{\prime}\right) \mathrm{d} s^{\prime} .
\end{gathered}
$$

This homogeneous set of linear equations (8) has nontrivial solutions only if

$$
\operatorname{det}\left(c_{m l}+\delta_{m l}\right)=0,
$$

where $\delta_{m l}$ is Kronecker's delta.

Solution in the limit of weak binding. We solve Eq. (5) with the boundary condition (8) as an expansion in $\lambda$

$$
f_{m}=\sum_{n=0}^{\infty} \lambda^{n} f_{m}^{(n)}, a_{m}=a_{0} \sum_{n=1}^{\infty} \lambda^{n} a_{m}^{(n)}
$$

where $a_{0}$ is a normalization constant multiplying all $a_{m}$ for $m \neq 0$. In the lowest order we find $a_{m}^{(1)}$ from Eq. (8) using $f_{0}^{(0)}(0, s)=\sqrt{s}$ for $s|\alpha| \ll 1$. Then Eq. (5) gives $f_{m}^{(0)}$ and $f_{0}^{(1)}$,

$$
\begin{gathered}
f_{0}^{(1)}(0, s)=-\sqrt{s} \int_{0}^{s} s^{\prime} V_{00}\left(s^{\prime}\right) \ln \left(s^{\prime} / s\right) \mathrm{d} s^{\prime} \\
f_{m}^{(0)}(0, s)=s^{|m|} \sqrt{s}+\frac{\int_{0}^{s} \frac{\left(s^{\prime}\right)^{2|m|}-(s)^{2|m|}}{\left(s s^{\prime}\right)|m|} s^{\prime} V_{m 0}\left(s^{\prime}\right) \mathrm{d} s^{\prime}}{\int_{0}^{\infty} V_{m 0}\left(s^{\prime}\right)\left(s^{\prime}\right)^{1-|m|} \cdot \mathrm{d} s^{\prime}} .
\end{gathered}
$$

Solving Eq. (10) in the leading order in $\lambda$ gives the energy

$$
\begin{gathered}
E=-\frac{2 \hbar^{2}}{\mu d^{2}} \exp \left(-2 \gamma-2 /\left(\lambda^{2} A_{0}\right)\right) \\
A_{0} \equiv-\int_{0}^{\infty} \sqrt{s} V_{00}(s) f_{0}^{(1)}(0, s) \mathrm{ds}+ \\
\sum_{m \neq 0} \int_{0}^{\infty} \frac{s^{1-|m|}}{2|m|} V_{m 0}(s) \mathrm{d} s \int_{0}^{\infty} \sqrt{s^{\prime}} V_{0 m}\left(s^{\prime}\right) f_{m}^{(0)}\left(0, s^{\prime}\right) \mathrm{d} s^{\prime},
\end{gathered}
$$

where $\gamma$ is the Euler's constant. Next order corrections can be determined iteratively using the calculated $a_{m}^{(1)}, f_{m}^{(0)}, f_{0}^{(1)}$ as described in [19]. The asymptotic form of the radial wavefunctions for $2 \mathrm{D}$ and finite-range anisotropic potentials is given generally as

$$
f_{m}(\alpha, s) \rightarrow \sqrt{s} H_{|m|}^{(1)}(\alpha s) \delta_{m 0},|\alpha| s \ll 1 .
$$

There is always a bound state for very weak potentials even for zero net volume, $\int r V \mathrm{~d} r \mathrm{~d} \varphi \leq 0$, and the threshold behavior of the energy is given by Eq. (14). For cylindrical potentials only the first term in Eq. (15) contributes as $V_{0 m} \propto \delta_{0 m}$, but even the leading order is still in general complicated.

Dipole results. Our first application is the system of two polarized molecules of reduced mass $\mu=M / 2$ confined to two parallel planes separated by a distance $d$. The corresponding dipole-dipole potential, $V$, projected to this two-dimensional geometry is

$$
V(r, \varphi)=D^{2} \frac{r^{2}+d^{2}-3(r \cos \varphi \cos \theta+d \sin \theta)^{2}}{\left(r^{2}+d^{2}\right)^{5 / 2}},
$$

where $D$ is the dipole moment and $\theta$ is the polarization angle measured from plane. This potential is reflection invariant with zero net volume for any polarization. It has monopole, dipole, and quadrupole terms only. The strength is now $\lambda \rightarrow U \equiv M D^{2} /\left(d \hbar^{2}\right)$, the non-zero matrix elements are $V_{m, m}, V_{m, m \pm 1}, V_{m, m \pm 2}$. Contributions to second order in $U$ on the right hand side of Eq. (14) are included by using $m=0, \pm 1, \pm 2$ since higher partial waves only contribute to the wave function through at least third order in $U$. We here systematically restrict ourselves to first order, and arrive at the energy expression

$$
E=-\frac{4 \hbar^{2}}{M d^{2}} \exp \left(-2 \gamma-\frac{2\left(1+U B_{1}\right)}{U^{2}\left(A_{0}+U A_{1}\right)}\right),
$$

where the coefficients, $A_{0}, A_{1}$ and $B_{1}$, are defined by

$$
\begin{aligned}
A_{0} & =\frac{1}{4} M_{c}^{2}+\frac{1}{8} \sin ^{2} 2 \theta+\frac{1}{32} \cos ^{4} \theta \\
A_{1} & =+0.0053 \sin ^{2} 2 \theta \cos ^{2} \theta-0.0033 \sin 2 \theta \cos ^{4} \theta \\
& -0.0019 \cos ^{6} \theta-M_{c}\left(0.0349 \sin ^{2}(2 \theta)\right. \\
& \left.+0.0054 \cos ^{4}(\theta)+0.0156 M_{c} \cos ^{2} \theta+0.0343 M_{c}^{2}\right) \\
B_{1} & =-1.204 M_{c}-\frac{1}{16} \cos ^{2} \theta \\
M_{c} & =\frac{3}{2} \sin ^{2}(\theta)-\frac{1}{2}, ; \frac{a}{4 d}=\exp \left(\frac{\left(1+U B_{1}\right)}{U^{2}\left(A_{0}+U A_{1}\right)}\right)(22)
\end{aligned}
$$

Here $a$ is the scattering length which is a function of the strength and the polarization angle [20]. The energy very close to threshold is exponential in $U^{2}$, as seen in Eq. (14), and determined by the polarization angle through $A_{0}$. The first order terms, $\left(A_{1}, B_{1}\right)$, in $U$ exhibit the difference in approach to threshold for the different signs of the strength, $U$. The second order terms, $\left(A_{2}, B_{2}\right)$, are necessary to get the correct $U$-independent pre-exponential factor in the energy. These higher order terms are sums of a large number of contributions 


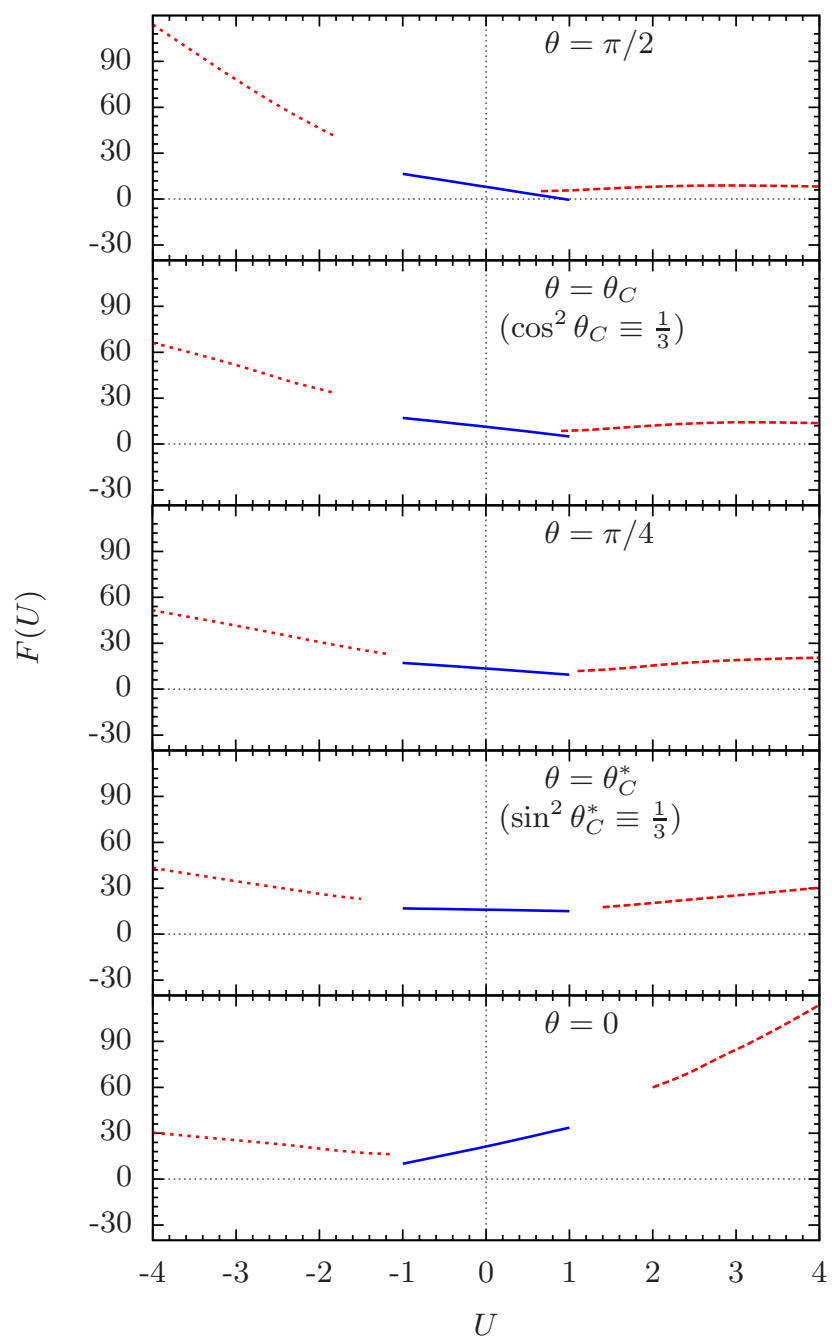

FIG. 1: The function $F(U) \equiv-U^{2} \ln \left(|E| M d^{2} /\left(2 \hbar^{2}\right)\right)$ for different polarization angles $\theta$. The dashed curves are calculated numerically and the solid lines are from Eq. (18).

expressed as definite integrals. The energy for $\theta=\pi / 2$ has been considered in recent studies 14 - 16]. While our result is in agreement with Ref. [16] to second order, the approximation in Ref. [15] deviates from our results in Eq. (18) in first order through the $A_{1}$-term. We give more details on the generalization to arbitrary polarization directions in [19].

Properties of weakly-bound states. The energy approaches zero extremely fast with vanishing strength, where the wave function only depends on binding energy through the modified Bessel function, $K_{0}(|\alpha| s)$. The rate of convergence towards these universal characteristics is less clear. We therefore designed a numerical method to investigate these structures, see [19] for more details.

The reduced energy, $\left|\alpha^{2}\right|$, is about $10^{-3}-10^{-6}$ when $U \simeq 1$, and the numerical results in Fig. 1 are not easily obtained due to the exponential square dependence on

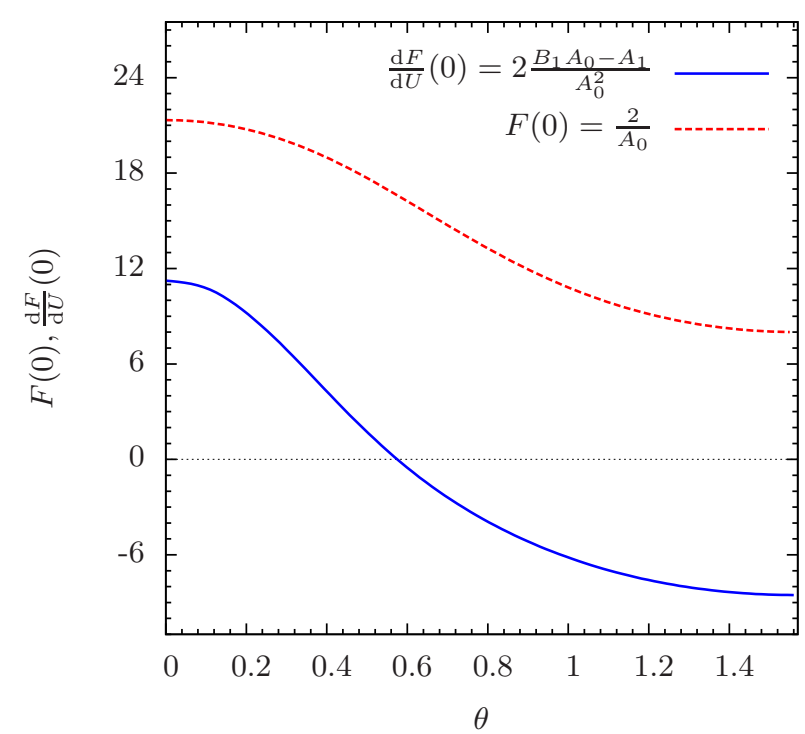

FIG. 2: The value (dashed) and the derivative (solid) with respect to $\mathrm{U}$ of the function, $F(U)=-U^{2} \ln \left(|E| M d^{2} /\left(2 \hbar^{2}\right)\right)$, for $U=0$ as functions of polarization angle.

$U$. We approach numerically the analytic straight line for small $U$, and find a very satisfying agreement for all $\theta$. We note that the omitted second order correction terms in Eq. (18) quickly, $U \simeq 0.5$, become significant in order to reproduce the parabolic behavior found numerically.

The binding energy is from Fig. 1 seen to increase or decrease with increasing $\theta$ for negative or positive $U$, repectively. This exponential variation with $\theta$, determined by $2 / A_{0}$, is shown in Fig. 2 in the limit $U \rightarrow 0$. The approach to $U=0$ also varies strongly with $\theta$ as illustrated in Fig. 2 by the slope which even changes sign at $\theta \approx 0.57$.

The structure of the wave function is revealed through the radial components, $f_{m}$, in Eq. (2). In the universal weak binding limit, $U \rightarrow 0$, the $m=0$ solution, $K_{0}$, is approached for all anisotropic potentials. This feature of universality is reached by smearing out the wave function over an ever increasing part of space. Outside of the potential, there emerges a avery small and slowly varying wave function whereas the contours of the potential are seen at smaller distances. This variation is found even for very small $U$, since the behavior near $r=0$ is essential to provide binding.

The convergence of the wave function towards $K_{0}$ implies that that the mean square radius in the limit is inversely proportional to the energy, $\left\langle r^{2}\right\rangle=2 \hbar^{2} /(3 M|E|)$, as shown in 20] and illustrated in Fig. 3. The limit is approached from above or below depending on the potential. A large barrier, (as for $U>0, \theta=\pi / 2$ ), confines the wave function to small distances even when the energy is relatively close to the threshold. However, eventually the wave function leaks out and the limit is approached from below. For $U<0, \theta \approx \pi / 2$ the barrier is absent and the approach for $E \rightarrow 0$ is from above. Approach 


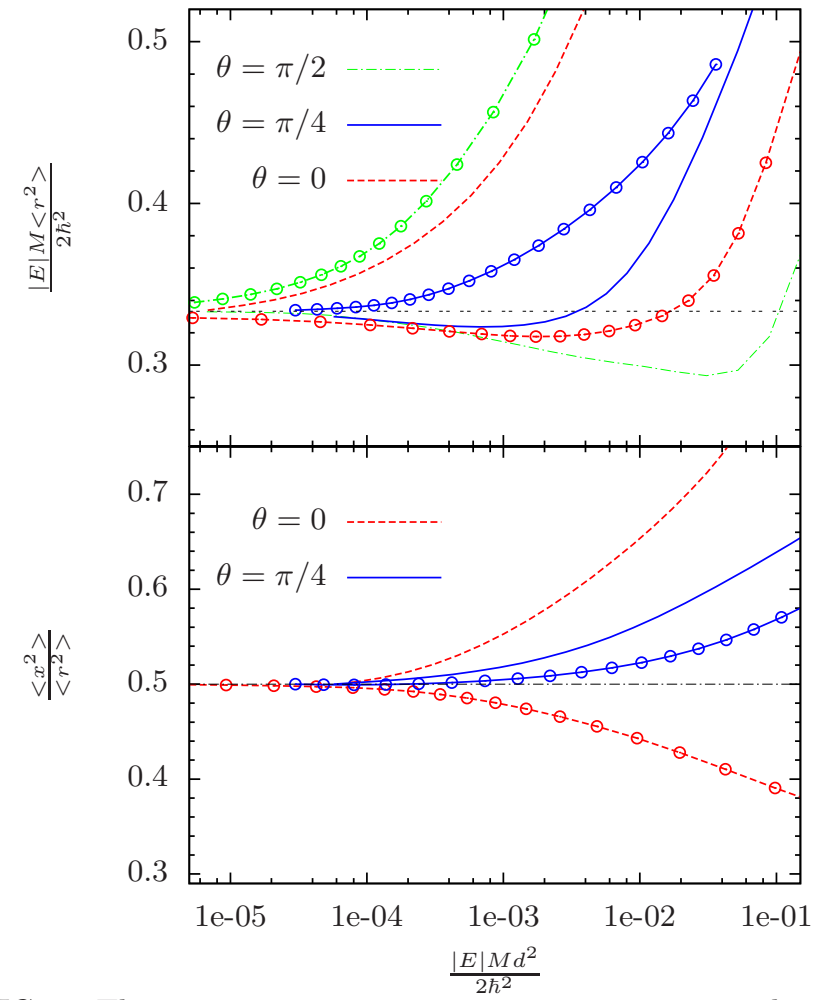

FIG. 3: The upper part is energy times mean square radius, $\left\langle r^{2}\right\rangle$. The lower part is the ratio of the second moment in the $x$-direction and $\left\langle r^{2}\right\rangle$ for different polarization angles $\theta$. The energy on the $x$-axis is obtained by varying the strength $U$ around zero, shown for $U<0$ and $U>0$ as curves with and without points, respectively. The horizontal line at $1 / 3$ is the universal limit.

to the universal limit for $E \rightarrow 0$ proves that only $m=0$ waves remain in analogy to halo nuclei in three dimensions. For much stronger binding $E\left\langle r^{2}\right\rangle$ increases as $E$ becomes large whereas $\left\langle r^{2}\right\rangle$ is confined by the radius of the attractive potential.

Another feature of universality is that the deviation from cylindrical symmetry on average disappears when $E \rightarrow 0$ as shown in Fig. 3. The rate of disappearance varies strongly with polarization angle. For $\theta=\pi / 2$ the potential itself is already cylindrical, whereas the largest deformation of potential and wave function occurs for $\theta=$ 0 . Increasing the angle results in less deformation for all energies. The elongation is largest along the polarization direction for all $U>0$. The deformations for $U<0$ are much smaller for comparable energies.

Perspectives. The two-body system has to be thoroughly understood to provide a solid ground for extraction of properties of the $N$-body system. We present an alternative proof that an arbitrary anisotropic $2 \mathrm{D}$ potential with zero net volume has at least one bound state for infinitesimal potential strength and rigorously derive a general expression for the energy for very weak potentials where the system reaches universality with a wave function given as a modified Bessel function entirely de- termined by the binding energy. The analytic result for the energy is worked out in details for dipolar molecules, and we illustrate the result by accurate numerical computations. High-order terms in the potential strength are necessary to get accurate values. We investigate characteristics of universality, that is approach to cylindrical symmetry, where the monopole component dominate and the mean square radius becomes inversely proportional to the binding energy. The universal limit is reached when the reduced strength, $|U|$, decreases below unity, which is the regime of current experiments [12].

These results imply that three particles in $2 \mathrm{D}$ without quantum statistical restrictions would have at least two bound states for weak couplings with universal features [20]. In conclusion, reliable predictions of many-body structures, including regions of various phases, must take these few-body structures into account as they persist to arbitrarily small potential strength.

[1] L. D. Landau and E. M. Lifshitz, Quantum Mechanics (Butterworth-Heinemann, Oxford, 1977).

[2] B. Simon, Ann. Phys. 97, 279 (1976).

[3] M. Combescure, C. Fayard, A. Khare, and J.-M. Richard, J. Phys. A. 44, 275302 (2011).

[4] J. Deslippe, C. D. Spataru, D. Prendergast, and S. G. Louie, Nano Lett. 7, 1626 (2007).

[5] D. G. Lidzey et al., Nature 395, 53 (1998).

[6] X.-Y. Zhu, Q. Yang, and M. Muntwiler, Acc. Chem. Res. 42, 1779 (2009).

[7] K. Miyake, Prog. Theor. Phys. 69, 1794 (1983); M. Randeria, J.-M. Duan, and L.-Y. Shieh, Phys. Rev. B 41, 327 (1990); D. S. Petrov, M. A. Baranov, and G. V. Shlyapnikov, Phys. Rev. A 67, 031601(R) (2003).

[8] F. E. Olsson et al., Phys. Rev. Lett. 93, 206803 (2004); L. Limot, E. Pehlke, J. Kröger, and R. Berndt, Phys. Rev. Lett. 94, 036805 (2005).

[9] S. Díaz-Tendero, A. G. Borisov and J. P. Gauyacq, Phys. Rev. B 76, 155428 (2007); B. Borca et al., Phys. Rev. Lett. 105, 036804 (2010).

[10] S. N. Coppersmith, R D. Blank, and L P. Kadanoff, J. Stat. Phys. 97, 429 (1999).

[11] M. A. Baranov, Phys. Rep. 464, 71 (2008); T. Lahaye, C. Menotti, L. Santos, M. Lewenstein, and T. Pfau, Rep. Prog. Phys. 72, 126401 (2009).

[12] M. H. G. de Miranda et al., Nature Physics 7, 502 (2011).

[13] G. M. Bruun and E. Taylor, Phys. Rev. Lett. 101, 245301 (2008); Y. Yamaguchi, T. Sogo, T. Ito, and T. Miyakawa, Phys. Rev. A 82, 013643 (2010); K. Sun, C. Wu, and S. Das Sarma, Phys. Rev. B 82, 075105 (2010); N. T. Zinner and G. M. Bruun, arXiv:1102.1551v1.

[14] J. R. Armstrong, N. T. Zinner, D. V. Fedorov, A. S. Jensen, Europhys. Lett. 91, 16001 (2010).

[15] M. Klawunn, A. Pikovski, and L. Santos, Phys. Rev. A 82, 044701 (2010).

[16] M. A. Baranov, A. Micheli, S. Ronen, and P. Zoller, Phys. Rev. A 83, 043602 (2011).

[17] E. Nielsen, D.V. Fedorov, A.S. Jensen, Few-Body Syst. 27, 15 (1999). 
[18] R.G. Newton, J. Math. Phys. 27 (1986) 2720.

[19] A. G. Volosniev, N. T. Zinner, D. V. Fedorov, A. S. Jensen, B. Wunsch, J. Phys. B. 44, 125301 (2011).
[20] E. Nielsen, D.V. Fedorov, A.S. Jensen, E. Garrido, Phys. Rep. 347, 373 (2001). 